\title{
Anatomic variation of alveolar antral artery
}

\author{
O.C. Ilie' ${ }^{1}$ R.C. Ciuluvică², M.C. Rusu² \\ ${ }^{1}$ Department of Anatomy, "Victor Babeş" University of Medicine and Pharmacy, Timişoara, Romania \\ 2Division of Anatomy, Faculty of Dental Medicine, "Carol Davila" University of Medicine and Pharmacy, \\ Bucharest, Romania
}

[Received 30 October 2014; Accepted 10 November 2014]

\begin{abstract}
The alveolar antral artery (AAA) was unanimously encountered in a few available studies with an intraosseous course to anastomose with the infraorbital artery. We report here two cases in which dissection revealed an extraosseous placement of this artery, between the lateral wall of the maxillary sinus and the Schneiderian membrane. The frequency of occurrence of the intraosseous anastomosis should be so modified from $100 \%$ to $<100 \%$. This arterial course over the Schneiderian membrane is important during surgical procedures: if it is identified preoperatively it can be avoided, or ligaturated, if not, it may be accidentally severed and uncomfortable haemorrhage may disturb the surgical procedure. In the first case reported here hybrid morphology of the AAA was also found, demonstrating that arterial anatomy should be considered with caution, on a case-by-case basis. (Folia Morphol 2015; 74, 2: 192-194)
\end{abstract}

Key words: maxillary sinus, alveolar artery, antrotomy, anatomic variation

\section{INTRODUCTION}

Knowledge of the vascular anatomy of the maxillary sinus (MS) ensures a good understanding of the vascular complication which can occur during various surgical procedures at this level [6]. The alveolar antral artery (AAA), which is the dental branch of the posterior superior alveolar artery (PSAA), anastomoses with the infraorbital artery (IOA) to form an anastomosis which was found intraosseous (endoosseous) in 100\% of cases $[4,6,9,10]$. Moreover, the gingival branch of the PSAA was found, but not always, anastomosed with the IOA by an extraosseous anastomosis covered by the oral mucosa $[6,9,10]$.

Here are reported two cases in which the so-considered intraosseous anastomosis of the PSAA and IOA was not, in fact, located within the MS wall, but was coursing beneath it, over the Schneiderian membrane.

\section{MATERIALS AND METHODS}

Intra-oral dissections were performed in 2 adult male cadavers of 68 and, respectively, 72 years old. In each case the outer wall of the oral vestibule was reflected and/or removed to access the MS outer walls. Mucosa was incised along the base of the alveolar process of the maxillary bone. Then the MS wall was cleared by en bloc removal of the soft tissues covering it. The sinus wall was trepanned and removed carefully to attempt keeping as much as possible undamaged the Schneiderian membrane.

\section{RESULTS}

In the first case (Fig. 1) the AAA was identified emerging from the PSAA and penetrating the MS wall at the lower end of the tuberosity of maxilla. After a short intraosseous course it continued between the Schneiderian membrane and the sinus wall, further

Address for correspondence: Prof. Dr. Hab. M.C. Rusu, "Carol Davila" University of Medicine and Pharmacy, 8 Bd. Eroilor Sanitari, RO-050474, Bucharest, Romania, tel: +40722363705, fax: +40213212284, e-mail: anatomon@gmail.com 


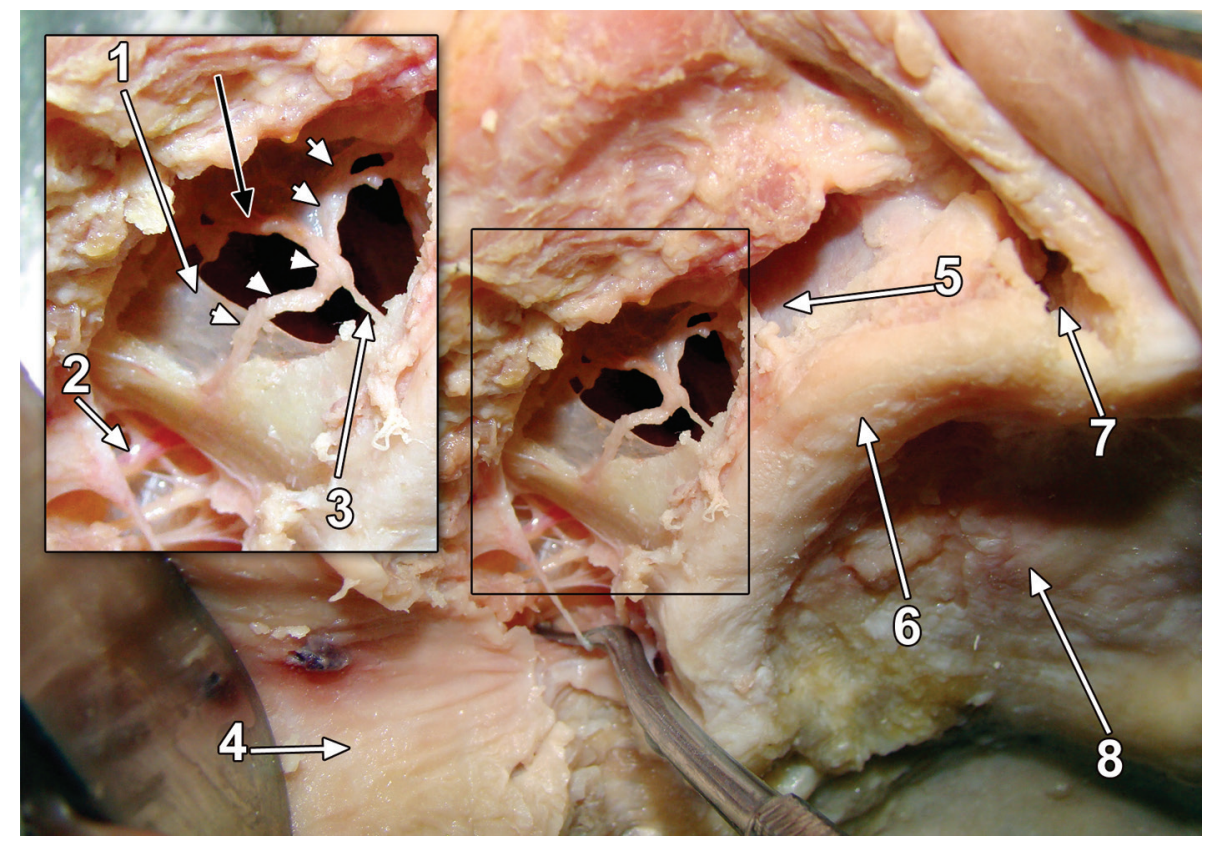

Figure 1. Antero-infero-lateral view of the right maxillary sinus (MS) walls, dissected by an intraoral approach. Unfixed specimen. The alveolar antral artery (AAA) is identified (arrowheads, zoomed-in inset) coursing between the MS wall and the Schneiderian membrane; 1 - Schneiderian membrane; 2 - posterior superior alveolar artery; 3 - secondary dental branch; 4 — buccal mucosa; 5 — Schneiderian membrane at the level of the canine fossa; 6 - upper edentulous maxillary arch; 7 - nasopalatine (incisive) canal; 8 - hard palate. The black arrow indicates a thick ascending branch of the AAA.

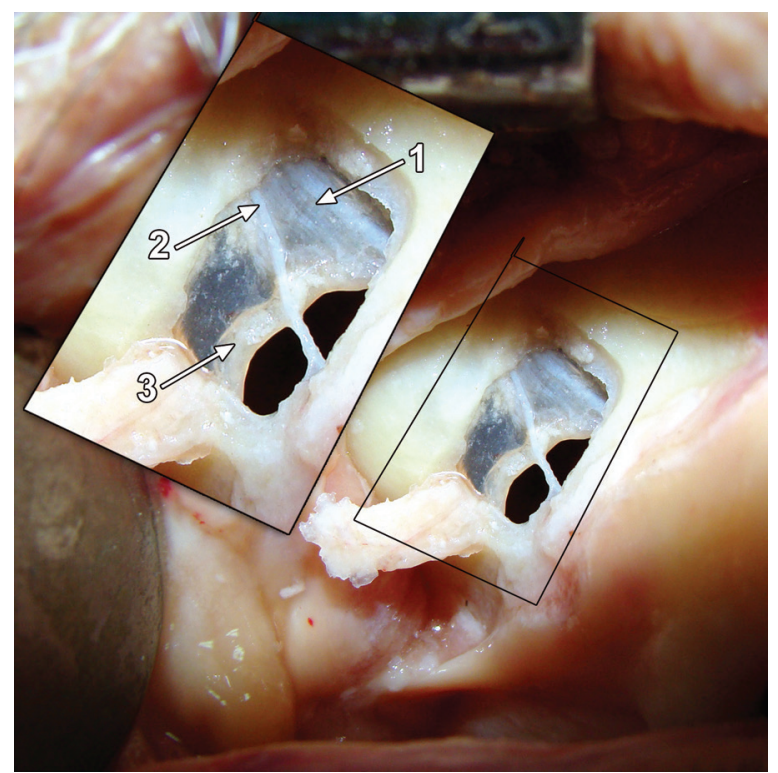

Figure 2. Lateral antrotomy of the right maxillary sinus (inset, zoomed-in) identifying beneath the sinus wall the Schneiderian membrane (1) crossed over by the alveolar antral artery (3) which, in turn, is crossed over by a superior dental nerve (2).

sending off descending dental branches but also an ascending one, towards the upper part of the posterior sinus wall.
In the second case (Fig. 2) only the antero-lateral wall of the MS trepanation was performed to simulate the sinus floor lift procedure. The bone window was reflected posteriorly carefully to complete the lateral antrotomy. The AAA was then found coursing on the Schneiderian membrane, being crossed over by a superior dental nerve which, according to its location above the upper premolars, was assumed being branched from the middle superior alveolar nerve.

\section{DISCUSSION}

As previous reports located the AAA exclusively within the MS wall (see Introduction) we assumed that this topographical variants we report here are rare or extremely rare. In this regard, practitioners should consider as $<100 \%$ the occurrence of the intraosseous anastomosis of the AAA and IOA.

Location of the AAA on the Schneiderian membrane is of great benefit but also of risk for surgeons, during the lateral antrotomy procedures. The risk would be if only an intraosseous location is assumed prior to the intervention, then the variant artery is encountered and damaged, leading to an unwanted haemorrhagic incident during the procedure. The 
benefit is determined by this particular location because, if the topographical variant is assumed as possible, surgical haemostasis could be safely performed.

We consider important prior to a surgical procedure to document the anatomy of the canals within the MS walls. In this regard, cone beam computed tomography (CBCT) was proven as the most reliable tool for the evaluation of the osseous anatomy of the mandible, or of maxillary bone, on a case-by-case basis [1, 3, 5]. Moreover, although several studies offered various measurements to support an adequate location of the $A A A, C B C T$ can do the same, patient by patient, except those situations, previously unreported, in which the AAA does not have an intraosseous canal to be identified in CBCT.

Dissections of the PSAA referred the initial course of the AAA to an horizontal line through the superior border of the infraorbital foramen and defined three patterns of this course, ascendant (pattern 1), slightly descendant (pattern 2) and with an abrupt descent (pattern 3) [11]. As referred to these anatomic possibilities which were found in Japanese cadavers, we found in case 1 a hybrid pattern: the dichotomized AAA sent off a superior branch with an ascending course (similar to pattern 1) and continued slightly descendant, like in pattern 2.

It was discussed the need for a realistic approach of the anatomical vascular structures [7] by keeping in mind the recommendation for not being seduced by the Vesalian anatomy [2], which is frequently overridden by unexpected anatomic variations [8], such as these reported here.

\section{CONCLUSIONS}

It appears that the intraosseous anastomosis of the AAA and IOA is not unanimously located within the maxillary sinus wall. Thus, the frequency of occurrence of the intraosseous course of that anasto- mosis should be considered being less than $100 \%$. As also hybrid morphologies of the AAA can occur, the anatomy of this artery should be considered on a case-by-case basis during specific surgical procedures.

\section{REFERENCES}

1. Apostolakis D, Brown JE (2013) The dimensions of the mandibular incisive canal and its spatial relationship to various anatomical landmarks of the mandible: a study using cone beam computed tomography. Int J Oral Maxillofac Implants, 28: 117-124.

2. Bergman RA (2011) Thoughts on human variations. Clin Anat, 24: 938-940.

3. Kapila S, Conley RS, Harrell WE, Jr. (2011) The current status of cone beam computed tomography imaging in orthodontics. Dentomaxillofac Radiol, 40: 24-34.

4. Kqiku L, Biblekaj R, Weiglein AH, Kqiku X, Stadtler P (2013) Arterial blood architecture of the maxillary sinus in dentate specimens. Croat Med J, 54: 180-184.

5. Parnia F, Moslehifard E, Hafezeqoran A, Mahboub F, Mojaver-Kahnamoui H (2012) Characteristics of anatomical landmarks in the mandibular interforaminal region: a cone-beam computed tomography study. Med Oral Patol Oral Cir Bucal, 17: e420-e425.

6. Rosano G, Taschieri S, Gaudy JF, Del Fabbro M (2009) Maxillary sinus vascularization: a cadaveric study. J Craniofac Surg, 20: 940-943.

7. Rusu MC, Pop E (2013) Fenestrated vertebral artery. Anat Sci Int, 88: 249-253.

8. Rusu MC, Vrapciu AD, Patrascu JM (2015) Variable relations of the trochlear nerve with the pontomesencephalic segment of the superior cerebellar artery. Surg Radiol Anat, 37: 555-559.

9. Solar P, Geyerhofer U, Traxler H, Windisch A, Ulm C, Watzek G (1999) Blood supply to the maxillary sinus relevant to sinus floor elevation procedures. Clin Oral Implants Res, 10: 34-44.

10. Traxler H, Windisch A, Geyerhofer U, Surd R, Solar P, Firbas W (1999) Arterial blood supply of the maxillary sinus. Clin Anat, 12: 417-421.

11. Yoshida S, Kawai T, Asaumi R, Miwa Y, Imura K, Koseki H, Sunohara M, Yosue T, Sato I (2010) Evaluation of the blood and nerve supply patterns in the molar region of the maxillary sinus in Japanese cadavers. Okajimas Folia Anat Jpn, 87: 129-133. 